\title{
Analysis of Chromatin Structure by Ligation-mediated PCR
}

\author{
Gerd P. Pfeifer
}

Beckman Research Institute of the City of Hope, Department of Biology, Duarte, California 91010

$\mathbf{T}_{\mathrm{n}}$ he expression of eukaryotic genes is mediated at the transcriptional level by a complex interplay between trans-acting factors and cis-acting elements. Interactions of proteins with specific DNA sequences are most commonly studied in vitro by applying the popular gel mobility-shift assays and footprinting techniques. These experiments are usually done using nuclear extracts from cell lines or tissues of interest. Beyond this type of analysis, it is desirable to have evidence that a protein which complexes with a particular DNA sequence in the test tube is actually bound to the same sequences under in vivo conditions. Within the cell, the accessibility of a sequence to a trans-acting factor may be restricted by a particular chromatin environment. Dynamic interactions of protein factors with their DNA target may be accompanied by changes in chromatin structure. These complex situations and processes are difficult to reproduce in vitro.

To investigate protein-DNA contacts in living cells, in vivo footprinting strategies have been devised. ${ }^{(1,2)}$ These methods are based on direct genomic sequencing ${ }^{(3)}$ where total genomic DNA is subjected to base-specific chemical cleavage and the sequence of a particular single-copy gene is then revealed without conventional cloning procedures. For complex genomes, such as those of mammals, special techniques are required to accomplish this goal. The most sensitive method uses the ligation-mediated polymerase chain reaction (LMPCR). ${ }^{(4-6)}$ This method significantly increases the sensitivity and specificity of the original genomic sequencing methods. All of the component fragments of a DNA sequence ladder are simultaneously amplified by PCR and displayed as sequencing gel autoradiograms. LMPCR preserves the quantitative representation of each fragment of the sequence ladder in the original population of cleaved molecules and each sequence ladder can be amplified very reproducibly. This has made possible its use for in vivo footprinting, ${ }^{(4)}$ genomic sequencing, ${ }^{(5)}$ methylation analysis, ${ }^{(5,7,8)}$ and studies on chromatin structure at single-nucleotide resolution. ${ }^{(9)}$

\section{LIGATION-MEDIATED PCR}

The starting material for LMPCR is total genomic DNA that has undergone a specific cleavage procedure to result in a DNA strand break or nick. Either chemical or enzymatic cleavage methods can be used. For analysis of chromatin structure in vivo, base modification, such as methylation of guanines with dimethylsulfate, or cleavage reactions, such as DNase I cleavage, have to be carried out within the cellular environment under conditions that retain the integrity of the analyzed structures. After DNA purification, gene-specific fragments are amplified from cleaved genomic DNA by LMPCR as follows (Fig. 1 summarizes the procedure; technical details of the method can be found in ref. 10).

The unique aspect of LMPCR is the ligation of an oligonucleotide sequence onto the 5' end of each DNA molecule. Because a phosphate group is required in this ligation reaction, it is advantageous to use a DNA cleavage method that leaves behind a 5' phosphate group on the sugar phosphate backbone. Cleavage of DNA to generate 5' phosphorylated molecules can be achieved, for example, by the $\beta$-elimination step of chemical DNA sequencing. ${ }^{(11)}$ Next, primer extension of a gene-specific oligonucleotide (primer 1) generates molecules that have a blunt end on one side. Linkers are li- gated to the blunt ends to introduce a common sequence to the 5' ends of the sequence ladder fragments. The linker has a $5^{\prime}$ overhang to prevent ligation in the wrong orientation. Then, an exponential PCR amplification of the linkerligated fragments is done using the longer oligonucleotide of the linker (linker-primer) and a second, nested gene-specific primer (primer 2).

After 15-20 cycles of PCR, the DNA fragments are separated on a sequencing gel, electroblotted onto nylon membranes, and hybridized with a gene-specific probe to visualize the sequence ladder. ${ }^{(5)}$ The single-stranded hybridization probes, which should not overlap the sequence of the second primer by more than 10 bases, can easily be made from an appropriate PCR product by repeated run-off polymerization with Taq polymerase using one of the two PCR primers. ${ }^{(10,12)}$ By rehybridization of the nylon membranes, several gene-specific ladders can be sequentially visualized from one sequencing gel (multiplexing). ${ }^{(5)}$ Alternatively, amplified fragments can be detected by primer extension using a ${ }^{32}$ P-labeled third primer. ${ }^{(4)}$ Only $1 \mu \mathrm{g}$ of mammalian DNA is required per lane of the sequencing gel to obtain good-quality DNA sequence ladders with retention of information on DNA cytosine methylation and in vivo protein-DNA contacts.

\section{LMPCR FOR ANALYSIS OF DNA METHYLATION PATTERNS}

It is now well established that methylation of 5'-CG dinucleotides within promoter regions of a gene correlates with a lack of gene expression. ${ }^{(13-16)}$ LMPCR has been used for sequencing genomic DNA and for determination of DNA cytosine methylation patterns. ${ }^{(5,7,8,17,18)}$ 
cleaved genomic DNA
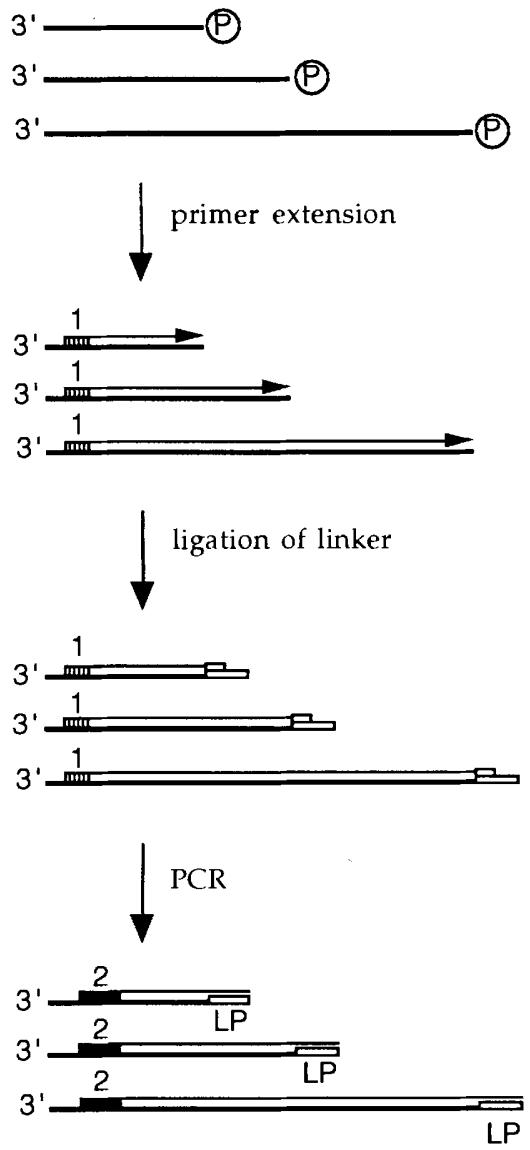

$\downarrow \begin{aligned} & \text { hybridization } \\ & \text { after gel transfer }\end{aligned}$

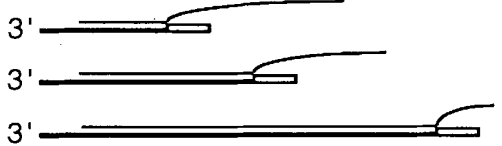

FIGURE 1 Outline of LMPCR. A gene-specific oligonucleotide (primer 1 ) is annealed to genomic DNA fragments that have been obtained by a specific cleavage method, e.g., DNase I treatment or chemical DNA sequencing. This primer is extended with a DNA polymerase to provide blunt ends for ligation of the common linker. Then, a second gene-specific primer (primer 2), which partially overlaps but extends $10-15$ bases 3 ' to primer 1 , is used together with the common linker primer (LP) in an exponential PCR reaction. The amplified fragments are separated on a sequencing gel and detected after blotting and hybridization with a single-stranded probe that does not overlap the sequence of primer 2 . Alternatively, the amplification products can be visualized by primer extension using a ${ }^{32} \mathrm{P}$-labeled, third gene-specific primer ${ }^{(4)}$ and autoradiography of the sequencing gel.
Methylated cytosines are recognized by their failure to react with hydrazine, thus resulting in a gap in the C-specific sequencing ladders. Another useful method for detecting 5-methylcytosine is based on UV-irradiation. 5-Methylcytosine, unlike cytosine, does not participate in formation of pyrimidine(6-4) pyrimidone photoproducts $[(6-4)$ photoproducts]. ${ }^{(19)}$ These UV-induced photoproducts occur predominantly at $5^{\prime}-\mathrm{TC}$ and $5^{\prime}$-CC dinucleotides. ${ }^{(20)}$ To detect (64) photoproducts at specific nucleotide positions within a gene, UV-irradiated DNA is cleaved at the sites of these photoadducts with hot piperidine, and genespecific fragments are then amplified by LMPCR. ${ }^{(21)}$ The absence of a (6-4) photoproduct at, for example, 5'-TCG and 5 '-CCG sequences indicates methylation of these sequences.

\section{GENOMIC FOOTPRINTING WITH DIMETHYLSULFATE}

Dimethylsulfate (DMS) was the first reagent to be used for in vivo footprinting studies $^{(22)}$ and is still the most commonly used one today. ${ }^{(23-30)}$ DMS is a small molecule that easily diffuses through cell membranes to react with DNA bases in the nucleus. 7-Methylguanines and 3-methyladenines are the predominant modified bases. After treating the cells with DMS, DNA is isolated and cleaved at 7-methylguanines with hot piperidine $^{(11)}$ or at 7-methylguanine and 3-methyladenine by heating at neutral $\mathrm{pH}$ followed by strand breakage in alkali. ${ }^{(28)}$ Gene-specific ladders are then amplified by LMPCR. ${ }^{(4,7)}$ Differences in the modification patterns between DMStreated cells and DMS-treated purified DNA, evident as hyperreactivities or protections, indicate in vivo protein-DNA contacts or an otherwise altered DNA structure at these sites.

The most significant advantage from using DMS as a footprinting agent is that it can be simply added to the cell culture medium; no manipulation of the cells is required. However, there are some disadvantages: (1) only sequences that contain Gs (or As) will reveal a footprint, (2) DMS can detect protein-DNA contacts only in the major groove of the DNA double-helix, (3) the reagent may penetrate protein-DNA complexes, and (4) since DMS readily reacts with proteins, only those protein-DNA interactions that remain stable after methylation of the protein factor itself will be indicated. In fact, we found that several footprints at the promoter of the human phosphoglycerate kinase (PGK1) gene were difficult to detect with DMS, but were more readily revealed by footprinting with DNase I or UV light. ${ }^{(9,31)}$

\section{GENOMIC FOOTPRINTING WITH DNASE I}

DNase I is much less base-selective than DMS and, in addition, the footprints from the $31-\mathrm{kD}$ DNase I enzyme are much larger and clearer than DMS footprints. These features have made DNase I the preferred agent for footprinting in vitro. Because of its size, DNase I cannot penetrate cell membranes. It can be used on isolated nuclei, but in many cases isolated nuclei do not retain all genuine protein-DNA contacts owing to a leakage of factors during nuclear isolation procedures. ${ }^{(9,32)}$ Zhang and Gralla recently demonstrated that SV40 minichromosomes in infected cells can be studied by DNase I and micrococcal nuclease treatment of lysolecithin-permeabilized cells. ${ }^{(33)}$ Cells permeabilized by lysolecithin remain intact, display very efficient DNA replication and transcription activities, ${ }^{(34,35)}$ and should maintain normal nuclear structure much better than isolated nuclei. We adapted LMPCR-aided genomic sequencing for studies of chromatin structure in permeabilized cells using DNase $\mathrm{I}^{(9)}$ and obtained very informative footprints at the promoter of the X-linked PGK1 gene. Footprints were seen only at the promoter of the active gene, but not when the gene was on the inactive $X$ chromosome. The inactive promoter showed enhanced DNase I cleavage sites that occurred with a periodicity of $10 \mathrm{bp}$ and spanned two nucleosome-size regions. Thus, genomic DNase I footprinting, coupled with LMPCR, proved to be a sensitive method for detecting sequencespecific transcription factors as well as positioned nucleosome-like particles. Figure 2 shows an example of DNase I footprints obtained from permeabilized cells by using LMPCR.

To amplify genomic fragments derived from DNase I cleavage efficiently, a modified LMPCR method was developed. DNase I cleavage results in fragments having $3^{\prime}$-hydroxyl groups. These fragments will contribute to high levels of nonspecific priming events during the 


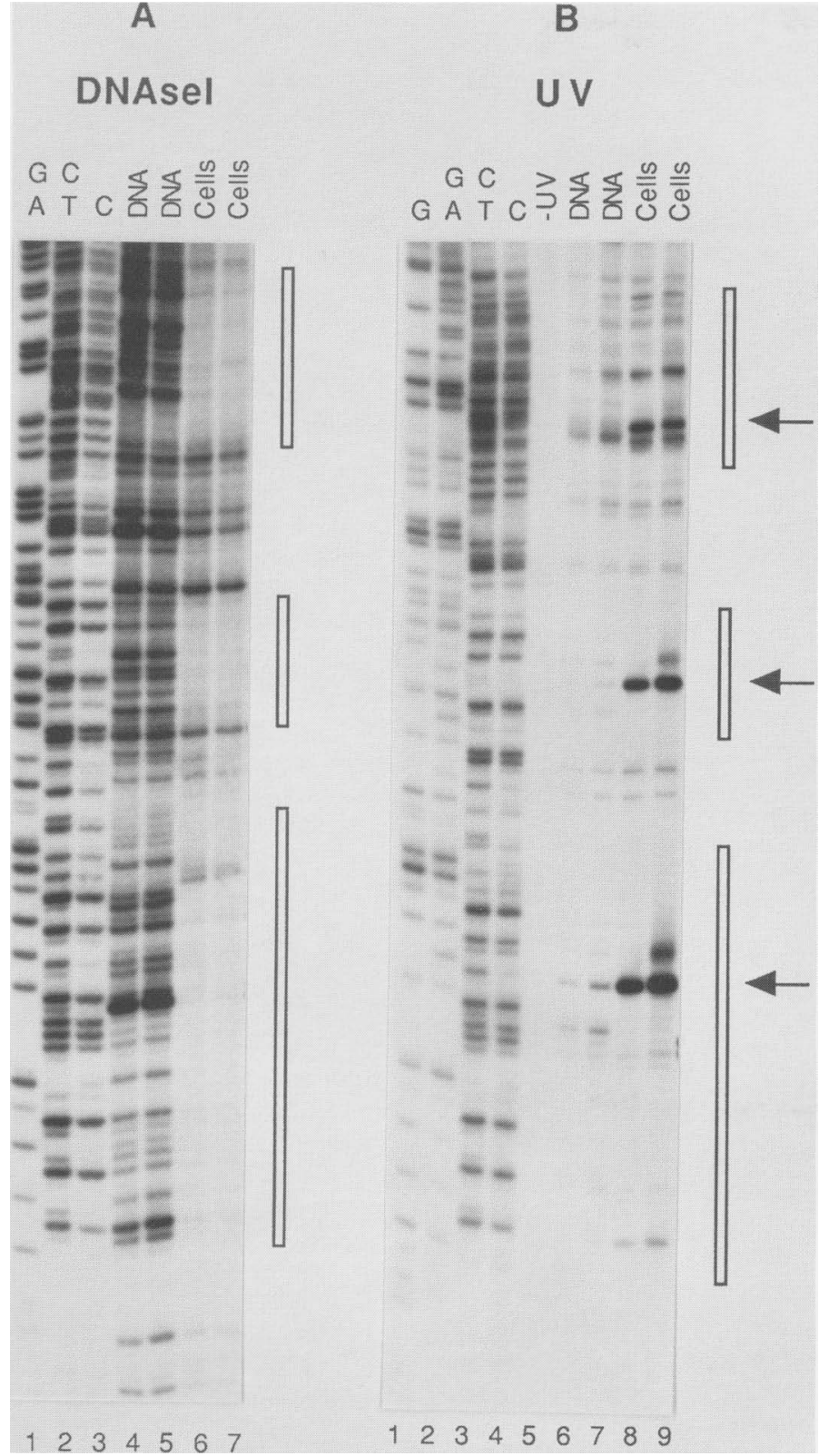

FIGURE 2 Examples of genomic DNase I footprinting and UV photofootprinting. (A) DNase I footprint analysis of the active $P G K 1$ promoter in permeabilized cells. ${ }^{(9)}$ Sequences are from the lower strand between nucleotides -30 and -170 . (Lanes 1-3) Maxam-Gilbert-cleaved DNA $(\mathrm{G}+\mathrm{A}, \mathrm{T}+\mathrm{C}, \mathrm{C})$ from HeLa cells; (lanes 4 and 5) DNase I-treated naked DNA controls from Y162 cells (containing the active PGK1 gene); (lanes 6 and 7) DNA from permeabilized Y162 cells treated with DNase I. DNase I footprints are indicated by the open boxes. $(B)$ UV footprint analysis of the $P G K 1$ promoter along the same sequences. ${ }^{(31)}$ (Lanes 1-4) Maxam-Gilbert sequencing reactions; (lane 5) DNA from Y162 cells, no UV irradiation; (lanes 6 and 7) distribution of cyclobutane dimers in UV-irradiated purified DNA; (lanes 8 and 9) distribution of dimers in UV-irradiated Y162 cells. Arrows indicate three dipyrimidine sequences that are strongly hyperreactive in vivo. Note that differences in photoreactivity coincide with DNase I footprints.

LMPCR reactions. This problem was initially circumvented by blocking the $3^{\prime}$ $\mathrm{OH}$ groups with a dideoxynucleotide using terminal deoxynucleotidyl transferase. ${ }^{(36)}$ More recently, we found that DNase I-cut genomic DNA can be used directly for LMPCR if fragments of interest are enriched by extension product capture. After extension of a biotinylated gene-specific primer with a DNA polymerase and ligation of the linker, specific fragments are bound to magnetic streptavidin-coated beads, and most of the genomic DNA is removed by washing the beads. Specifically bound fragments are eluted from the beads in sodium hydroxide and amplified by PCR with a second gene-specific primer and the linker primer. This procedure reduces nonspecific background and improves the clarity of the sequence ladders. The improvements are particularly significant for DNase I-treated DNA samples because most of the small genomic DNA fragments that may contribute to nonspecific priming and amplification events are eliminated prior to the PCR reactions (V.T. Törmänen, G.P. Pfeifer, and A.D. Riggs, in prep.).

\section{IN VIVO FOOTPRINTING WITH UV LIGHT}

The use of ultraviolet (UV) light as an agent to detect protein-DNA complexes was pioneered by Becker and Wang. ${ }^{(37)}$ Differences in UV reactivity between irradiated purified DNA and irradiated protein-DNA complexes are referred to as "photofootprints." UV irradiation is probably the least disruptive method to detect DNA-bound proteins in vivo, because intact cells in a Petri dish are simply irradiated from a UV light source for a short period of time. No complex manipulations are necessary.

UV irradiation of DNA produces, in order of their relative frequency, (1) cyclobutane pyrimidine dimers, (2) (6-4) photoproducts, and (3) minor photoproducts, like cytosine hydrates and purine dimers. ${ }^{(20,38)}$ Cyclobutane dimers are formed between the 5,6 bonds of any two adjacent pyrimidines. (6-4) Photoproducts are characterized by a covalent bond between positions 6 and 4 of two adjacent pyrimidines, mostly between $5^{\prime}$-TpC and 5'-CpC. ${ }^{(20)}$ (6-4) Photoproducts in total genomic DNA are formed at a rate of $10-30 \%$ of that of cyclobutane dimers, ${ }^{(39)}$ but the relative abundance of the two photoproducts strongly depends on the local DNA sequence. LMPCR provides an adequate level of sensitivity for the detection of individual UV-induced DNA photoproducts at the DNA sequence level in mammalian DNA. ${ }^{(21)}$

Initially, LMPCR has been used for detection of (6-4) photoproducts. (6-4) Photoproducts and their Dewar isomers are sensitive to alkaline cleavage and can be assayed as piperidine labile sites. ${ }^{(40)}$ Piperidine cleavage of (6-4) photoprod- 
ucts results in breaks with a $5^{\prime}$-phosphate group that allows direct amplification by LMPCR. ${ }^{(21)}$ We also developed a method for mapping of cyclobutane pyrimidine dimers at nucleotide resolution. ${ }^{(31)}$ Cyclobutane dimers are resistant to alkaline cleavage, but can be detected at the DNA sequence level by cleavage with the cyclobutane dimerspecific endonuclease T4 endonuclease $V{ }^{(41)}$ This enzyme breaks the glycosylic bond and sugar phosphate backbone at cyclobutane dimers but does not cleave (6-4) photoproducts. Cleavage with T4 endonuclease $V$ leaves behind a dimerized pyrimidine at the $5^{\prime}$ end of the damaged strand. ${ }^{(42,43)}$ These fragments cannot be amplified by the LMPCR procedure directly but require further treatment with Escherichia coli photolyase $^{(44)}$ to split the overhanging pyrimidine dimers at the $5^{\prime}$ ends. ${ }^{(31)}$ The photolyase splitting reaction is complete and sequence-independent, which allows precise mapping of dimer frequencies along any DNA sequence.

Photoproducts in irradiated, purified genomic DNA are distributed with a pattern that is expected from experiments that have used cloned, end-labeled DNA for UV irradiation. ${ }^{(19,41,45,46)}(6-4)$ Photoproducts are seen preferentially at $5^{\prime}$ TC and 5'-CC sequences and cyclobutane dimers are induced in order of their relative frequency at $5^{\prime}-\mathrm{TT}>5^{\prime}-\mathrm{CT}=5^{\prime}$ TC $>5^{\prime}$-CC. However, when the photoproduct spectrum of irradiated purified DNA was compared with that obtained after irradiation of living cells, some striking differences became apparent. ${ }^{(31)}$ The photoproduct frequency within sequences bound by sequence-specific DNA-binding proteins (transcription factors) was suppressed or enhanced relative to naked DNA or inactive genes, which do not bind these factors. Figure 2 shows an example of these photofootprints displayed at the DNA sequence level by LMPCR mapping. The sequences shown are from the promoter region of the human single-copy gene PGK1. It is important to note that the differences in photoreactivity between irradiated purified DNA and irradiated cells fall within the same sequences protected from DNase I in permeabilized cells (Fig. 2). Thus, photofootprints are reliable indicators of sequence-specific protein-DNA contacts in vivo. The most conspicuous differences between irradiated cells and irradiated purified DNA were transcrip- tion factor-induced photoproduct hot spots within the promoter of the active gene. For example, the dinucleotide sequence 5 '-CC within the sequence CCAAT, the binding site for a CCAATbox binding protein, shows a (6-4) photoproduct signal 30-fold higher when cells with an active PGK1 gene were irradiated than when purified DNA or cells with an inactive PGK1 gene were irradiated. ${ }^{(31)}$ Effects of chromatin structure on UV damage may be most significant in regulatory gene regions as a consequence of binding of transcription factors. In other experiments, the frequency of cyclobutane dimers and (6-4) photoproducts was mapped along exons 1 and 2 of human ras proto-oncogenes. ${ }^{(12)}$ The photoproduct spectra were almost identical between irradiated purified DNA and irradiated cells, indicating absence of UV-detectable DNA binding proteins along the ras exon sequences. The theoretical basis for the occurrence of photofootprints has not been clearly defined. After local DNA sequences absorb a UV photon, only those excited bases in a geometry capable of easily forming a photoproduct can photoreact. Adjacent pyrimidines must rotate extensively from their usual B-form DNA alignments to form interpyrimidine covalent bonds. It is conceivable that the bound protein induces an alteration in DNA structure, possibly unwinding or bending, that is favorable or unfavorable for dimer formation. ${ }^{(37)}$ Mapping of cyclobutane dimers at the single-copy gene level may also indicate positioned nucleosomes since cyclobutane dimers are modulated with a 10-bp periodicity within nucleosome core DNA. ${ }^{(47,48)}$

\section{CONCLUSIONS}

LMPCR provides the necessary sensitivity for studies on chromatin structure at nucleotide resolution. When combined with an appropriate in situ footprinting technique, high-resolution mapping of protein-DNA contacts in mammalian cells becomes possible. There are at least three different approaches that can be used for LMPCR-footprinting: treatment of intact cells with DMS, probing permeabilized cells with DNase I, and UV irradiation of intact cells. One or the other method may be more suitable for a particular biological problem. For example, pyrimidine-rich sequences will not give very informative DMS footprints and purine-rich sequences cannot easily be analyzed with UV light. The most versatile method, although technically slightly more complex than the others, promises to be DNase I footprinting of permeabilized cells. In many cases, the combined use of these methods may provide the most informative and complete picture.

\section{ACKNOWLEDGMENTS}

I would like to thank G. Holmquist, A. Riggs, P. Szabó, and M. Hershkovitz for discussions and comments on the manuscript.

\section{REFERENCES}

1. Cartwright, I.L. and S.E. Kelly. 1991. Probing the nature of chromosomal DNA-protein contacts by in vivo footprinting. Biotechniques 11: 188-203.

2. Saluz, H.P., K. Wiebauer, and A. Wallace. 1991. Studying DNA modifications and DNA-protein interactions in vivo. Trends Genet. 7: 207-211.

3. Church, G.M. and W. Gilbert. 1984. Genomic sequencing. Proc. Natl. Acad. Sci. 81: 1991-1995.

4. Mueller, P.R. and B. Wold. 1989. In vivo footprinting of a muscle specific enhancer by ligation mediated PCR. Science 246: 780-786.

5. Pfeifer, G.P., S.D. Steigerwald, P.R. Mueller, B. Wold, and A.D. Riggs. 1989. Genomic sequencing and methylation analysis by ligation mediated PCR. Science 246: 810-813.

6. Garrity, P.A. and B.A. Wold. 1992. Effects of different DNA polymerases in ligationmediated PCR: Enhanced genomic sequencing and in vivo footprinting. Proc. Natl. Acad. Sci. 89: 1021-1025.

7. Pfeifer, G.P., R.L. Tanguay, S.D. Steigerwald, and A.D. Riggs. 1990. In vivo footprint and methylation analysis by PCRaided genomic sequencing: Comparison of active and inactive $X$ chromosomal DNA at the CpG island and promoter of human PGK-1. Genes \& Dev. 4: 12771287.

8. Pfeifer, G.P., S.D. Steigerwald, R.S. Hansen, S.M. Gartler, and A.D. Riggs. 1990. Polymerase chain reaction aided genomic sequencing of an $\mathrm{X}$ chromosomelinked $\mathrm{CpG}$ island: Methylation patterns suggest clonal inheritance, $\mathrm{CpG}$ site autonomy, and an explanation of activity state stability. Proc. Natl. Acad. Sci. 87: 8252-8256.

9. Pfeifer, G.P. and A.D. Riggs. 1991. Chromatin differences between active and inactive $\mathrm{X}$ chromosomes revealed by genomic footprinting of permeabilized cells 
using DNase I and ligation-mediated PCR. Genes \& Dev. 5: 1102-1113.

10. Pfeifer, G.P. and A.D. Riggs. 1992. Genomic footprinting by ligation mediated polymerase chain reaction. In Methods in Molecular Biology, vol. 16, PCR Protocols: Current Methods and Applications (ed. B.A. White), Humana Press, Totowa, NJ (in press).

11. Maxam, A.M. and W. Gilbert. 1980. Sequencing end-labeled DNA with base-specific chemical cleavages. Methods Enzymol. 65: 499-560.

12. Törmänen, V.T. and G.P. Pfeifer. 1992. Mapping of UV photoproducts within ras proto-oncogenes in UV-irradiated cells: Correlation with mutations in human skin cancer. Oncogene 7: 1729-1736.

13. Becker, P.B., S. Ruppert, and G. Schütz. 1987. Genomic footprinting reveals celltype specific DNA binding of ubiquitous factors. Cell 51: 435-445.

14. Cedar, H. 1988. DNA methylation and gene activity. Cell 53: 3-4.

15. Bird, A.P. 1992. The essentials of DNA methylation. Cell 70: 5-8.

16. Riggs, A.D. and G.P. Pfeifer. 1992. X chromosome inactivation and cell memory. Trends Genet. 8: 169-174.

17. Rideout III, W.M., G.A. Coetzee, A.F. Olumi, and P.A. Jones. 1990. 5-Methylcytosine as an endogenous mutagen in the human LDL receptor and p53 genes. Science 249: 1288-1290.

18. Sørenson, M.B. 1992. Methylation of B-hordein genes in barley endosperm is inversely correlated with gene activity and affected by the regulatory gene Lys3. Proc. Natl. Acad. Sci. 89: 4119-4123.

19. Glickman, B.W., R.M. Schaaper, W.A. Haseltine, R.L. Dunn, and D.E. Brash. 1986. The C-C (6-4) UV photoproduct is mutagenic in Escherichia coli. Proc. Natl. Acad. Sci. 83: 6945-6949.

20. Mitchell, D.L. and R.S. Nairn. 1989. The biology of the (6-4) photoproduct. Photochem. Photobiol. 49: 805-819.

21. Pfeifer, G.P., R. Drouin, A.D. Riggs, and G.P. Holmquist. 1991. In vivo mapping of a DNA adduct at nucleotide resolution: Detection of pyrimidine (6-4) pyrimidone photoproducts by ligation-mediated polymerase chain reaction. Proc. Natl. Acad. Sci. 88: 1374-1378.

22. Ephrussi, A., G.M. Church, S. Tonegawa, and W. Gilbert. 1985. B lineage specific interactions of an immunoglobulin enhancer with cellular factors in vivo. Science 227: 134-140.

23. Ikuta, T. and Y.W. Kan. 1991. In vivo protein-DNA interactions at the $\beta$-globin gene locus. Proc. Natl. Acad. Sci. 88: 10188-10192.

24. Kara, K.J. and L.H. Glimcher. 1991. In vivo footprinting of MHC class II genes: Bare promoters in the bare lymphocyte syndrome. Science 252: 709-712.
25. Reddy, P.M.S. and C.K.J. Shen. 1991. Protein-DNA interactions in vivo of an erythroid-specific, human $\beta$-globin locus enhancer. Proc. Natl. Acad. Sci. 88: 86768680.

26. Rigaud, G., J. Roux, R. Pictet, and T. Grange. 1991. In vivo footprinting of rat TAT gene: Dynamic interplay between the glucocorticoid receptor and a liverspecific factor. Cell 67: 977-986.

27. Tsai, S.F., E. Strauss, and S.H. Orkin. 1991. Functional analysis and in vivo footprinting implicate the erythroid transcription factor GATA-1 as a positive regulator of its own promoter. Genes \& Dev. 5: 919-931.

28. Strauss, E.C., N.C. Andrews, D.R. Higgs, and S.H. Orkin. 1992. In vivo footprinting of the human alpha-globin locus upstream regulatory element by guanine and adenine ligation-mediated polymerase chain reaction. Mol. Cell. Biol. 12: 2135-2142.

29. König, H., H. Ponta, H.J. Rahmsdorf, and P. Herrlich. 1992. Interference between pathway-specific transcription factors: Glucocorticoids antagonize phorbol ester-induced AP-1 activity without altering AP-1 site occupation in vivo. EMBO J. 11: 2241-2246.

30. Jurivich, D.A., L. Sistonen, R.A. Kroes, and R.I. Morimoto. 1992. Effect of sodium salicylate on the human heat shock response. Science 255: 1243-1246.

31. Pfeifer, G.P., R. Drouin, A.D. Riggs, and G.P. Holmquist. 1992. Binding of transcription factors creates hot spots for UV photoproducts in vivo. Mol. Cell. Biol. 12: 1798-1804.

32. Zhang, L. and J.D. Gralla. 1990. In situ nucleoprotein structure involving originproximal SV40 DNA control elements. Nucleic Acids Res. 18: 1797-1803.

33. Zhang, L. and J.D. Gralla. 1989. In situ nucleoprotein structure at the SV40 major late promoter: Melted and wrapped DNA flank the start site. Genes \& Dev. 3: 1814 1822.

34. Miller, M.R., J.J. Castellot, and A.B. Pardee. 1978. A permeable animal cell preparation for studying macromolecular synthesis. DNA synthesis and the role of deoxyribonucleotides in $S$ phase initiation. Biochemistry 17: 1073-1080.

35. Contreras, R. and W. Fiers. 1981. Initiation of transcription by RNA polymerase II in permeable SV40-infected CV-1 cells; evidence of multiple promoters for SV40 late transcription. Nucleic Acids Res. 9: 215-236.

36. Tanguay, R.L., G.P. Pfeifer, and A.D. Riggs. 1990. PCR-aided DNase I footprinting of single-copy gene sequences in permeabilized cells. Nucleic Acids Res. 18: 5902.

37. Becker, M.M. and J.C. Wang. 1984. Use of light for footprinting DNA in vivo. Nature 309: $682-687$.

38. Brash, D.E. 1988. UV mutagenic photo- products in Escherichia coli and human cells: A molecular genetics perspective on human skin cancer. Photochem. Photobiol. 48: 59-66.

39. Mitchell, D.L., D.E. Brash, and R.S. Nairn. 1990. Rapid repair kinetics of pyrimidine(6-4) pyrimidone photoproducts in human cells are due to excision repair rather than conformational change. $\mathrm{Nu}$ cleic Acids Res. 18: 963-971.

40. Lippke, J.A., L.K. Gordon, D.E. Brash, and W.A. Haseltine. 1981. Distribution of UV light-induced damage in a defined sequence of human DNA: Detection of alkaline-sensitive lesions at pyrimidine nucleoside-cytidine sequences. Proc. Natl. Acad. Sci. 78: 3388-3392.

41. Gordon, L.K. and W.A. Haseltine. 1980. Comparison of the cleavage of pyrimidine dimers by the bacteriophage T4 and Micrococcus luteus UV-specific endonucleases. J. Biol. Chem. 255: 12047-12050.

42. Haseltine, W.A., L.K. Gordon, C.P. Lindan, R.H. Grafstrom, L.N. Shaper, and L. Grossman. 1980. Cleavage of pyrimidine dimers in specific DNA sequences by a pyrimidine dimer DNA-glycosylase of $M$. luteus. Nature 285: 634-641.

43. Grossman, L., P.R. Caron, S.J. Mazur, and E.Y. Oh. 1988. Repair of DNA containing pyrimidine dimers. FASEB I. 2: 26962701.

44. Sancar, A., F.W. Smith, and G.B. Sancar. 1984. Purification of Escherichia coli DNA photolyase. J. Biol. Chem. 259: 60286032.

45. Brash, D.E. and W.A. Haseltine. 1982. UVinduced mutation hotspots occur at DNA damage hotspots. Nature 298: 189-192.

46. Bourre, F., G. Renault, and A. Sarasin. 1987. Sequence effect on alkali-sensitive sites in UV-irradiated SV40 DNA. Nucleic Acids Res. 15: 8861-8875.

47. Gale, J.M., K.A. Nissen, and M.J. Smerdon. 1987. UV-induced formation of pyrimidine dimers in nucleosome core DNA is strongly modulated with a period of 10.3 bases. Proc. Natl. Acad. Sci. 84: 6644-6648.

48. Smerdon, M.J. 1991. DNA repair and the role of chromatin structure. Curr. Opin. Cell Biol. 3: 422-428. 


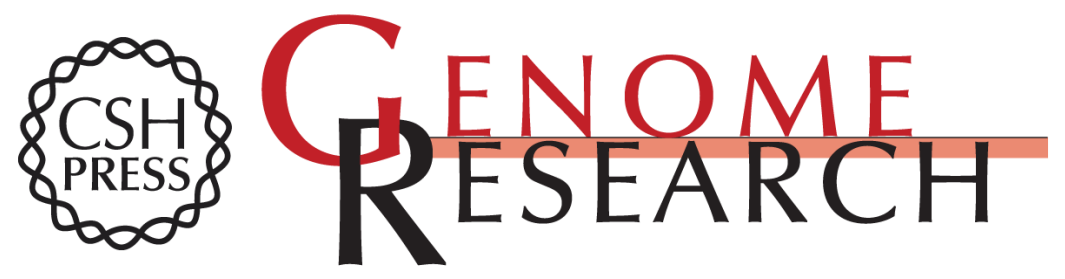

\title{
Analysis of chromatin structure by ligation-mediated PCR.
}

\author{
G P Pfeifer
}

Genome Res. 1992 2: 107-111

Access the most recent version at doi:10.1101/gr.2.2.107

References This article cites 47 articles, 23 of which can be accessed free at:

http://genome.cshlp.org/content/2/2/107.full.html\#ref-list-1

\section{License}

Email Alerting Receive free email alerts when new articles cite this article - sign up in the box at the Service top right corner of the article or click here.

\section{Affordable, Accurate Sequencing.}

To subscribe to Genome Research go to: https://genome.cshlp.org/subscriptions 